\title{
Experimental evidence of a cluster-glass transition on the colossal magnetoresistance manganite $\mathrm{La}_{0.7} \mathrm{~Pb}_{0.3}\left(\mathrm{Mn}_{0.9} \mathrm{Fe}_{0.1}\right) \mathrm{O}_{3}$
}

\author{
N. Veglio* and F. J. Bermejo ${ }^{\dagger}$ \\ Department of Electricity and Electronics, University of the Basque Country, P.O. Box 644, E-48080, Bilbao, Spain \\ J. Gutierrez, J. M. Barandiarán, and A. Peña \\ Department of Electricity and Electronics and Inorganic Chemistry, University of the Basque Country, P.O. Box 644, \\ E-48080, Bilbao, Spain \\ M. A. González, P. P. Romano, and C. Mondelli \\ Institut Laue Langevin, 6 Rue Jules Horowitz, BP 156 Grenoble Cedex 9, F-38042, Grenoble Cedex 9, France
}

(Received 2 August 2004; revised manuscript received 13 January 2005; published 6 June 2005)

\begin{abstract}
Neutron small angle scattering on a colossal magnetoresistance material shows clear signatures of a rise in characteristic length of paramagnetic fluctuations as the maximum of dc susceptibility is approached from high temperatures. The phenomenon is accompanied by a rise in intensity of a broad peak that appears at wave vectors of $Q \approx 0.025 \AA^{-1}$, and this is interpreted as a fingerprint of the onset of ferromagnetic ordering due to intercluster magnetic interactions.
\end{abstract}

DOI: 10.1103/PhysRevB.71.212402

PACS number(s): 75.40.-s, 75.47. $-\mathrm{m}, 61.12 . \mathrm{Ex}, 61.46 .+\mathrm{w}$

One of the most widely accepted scenarios to understand the colossal magnetoresistance (CMR) effect pictures coexisting clusters of two competing phases. ${ }^{1}$ Ferromagnetic nanoclusters, which above a critical temperature remain with their magnetic moments randomly oriented, react quickly to small applied fields yielding a preferential orientation of their moments. In the absence of applied fields, the competing (charge-ordered and/or antiferromagnetic) phase generates domain walls that prevent such an alignment. The search for such magnetic (or structural) inhomogeneities continues to focus a good number of efforts, ${ }^{2-4}$ reporting on inhomogeneities with sizes spanning from nano- to submicrometers. ${ }^{3}$ Also, dynamic correlates of such inhomogeneities are provided by results on time-dependent fluctuations ${ }^{5}$ which also point towards nanoscale phenomena.

Some of the experimental evidence brought forward in support for the presence of such inhomogeneities has at times relied upon analysis of the small-angle-scattering patterns $^{6,7}$ as measured with or without energy analysis, which is usually performed after subtraction of a paramagnetic, high-temperature contribution.

Here we report on recent findings concerning the mesoscopic structure of the iron-doped manganite $\mathrm{La}_{0.7} \mathrm{~Pb}_{0.3}\left(\mathrm{Mn}_{0.9} \mathrm{Fe}_{0.1}\right) \mathrm{O}_{3}$. Particular interest in this material stems from its large magnetoresistance ${ }^{8}$ while keeping its ground ferromagnetic state not largely perturbed by the addition of the transition metal ion. The latter will couple antiferromagnetically to nearest neighboring magnetic ions while manganese ions may either couple ferro- or antiferromagnetically depending upon the valences and nature of the intervening ions. For higher concentrations of the dopant this leads to significant frustration of the spin system since Mn ions will couple by both antiferromagnetic superexchange and double exchange mechanisms to neighboring atoms.

Previous neutron diffraction work $^{8}$ depicts the crystal structure of this family of compounds as trigonal (space group $R \overline{3} c)$ with $Z=6$, with La atoms at $(0,0,1 / 4)$, Mn at $(0,0,0)$ and $\mathrm{O}$ at $(x, 0,1 / 4)$ positions. Substitution of $\mathrm{Mn}$ by $\mathrm{Fe}$ induces a rather small distortion of the $\mathrm{MnO}_{6}$ octahedra which, in turn, is weakly dependent on the doping ratio. Long-range magnetic order is attained for the parent (undoped) and $x=0.1$ compounds below a main paramagnetic $\rightarrow$ ferromagnetic transition [see Fig. 2(a)] that shifts from $345 \mathrm{~K}$ for $x=0$ to $230 \mathrm{~K}$ for $x=0.1$, with a concomitant $15 \%$ reduction of magnetic moment which attains a value of $2.79 \mu_{\mathrm{B}}$. Increasing the Fe percentage up to $x=0.2$ withers away all signatures of magnetic long-range order, while keeping signatures of magnetic short-range order ${ }^{9}$ and behaving macroscopically as a complex magnet having both antiferromagnetic and ferromagnetic components. Indications of such competing magnetic interactions are also reavealed for the $x=0.1$ composition in a Mössbauer study on the Sr substitute isomorph. ${ }^{10}$ As a matter of fact, such a study reports on the coexistence of ferromagnetic regions together with either short-range-ordered or paramagnetic regions even within the magnetically ordered phase.

Raw data as measured for the $x=0.1$ compound on the low-to-middle angles diffractometer D16 at the Institut Laue Langevin for fine powdered samples were corrected for detector efficiency, background, multiple scattering, and selfabsorption, and normalized to an absolute scale using the Correct software package. ${ }^{11}$ The evolution with temperature of the measured single-differential cross section is shown in Fig. 1.

The most remarkable features appearing in the figure just referred to concern the development of a large paramagneticlike contribution at large wave vectors as well as the presence of a broad maximum at $Q \approx 0.025 \AA$. Also notice the crosssing over of curves within the range $0.03 \AA^{-1} \leqslant Q$ $\leqslant 0.06 \AA^{-1}$.

A model-free evaluation of the information content of data shown in Fig. 1 is provided by plots of the temperature 


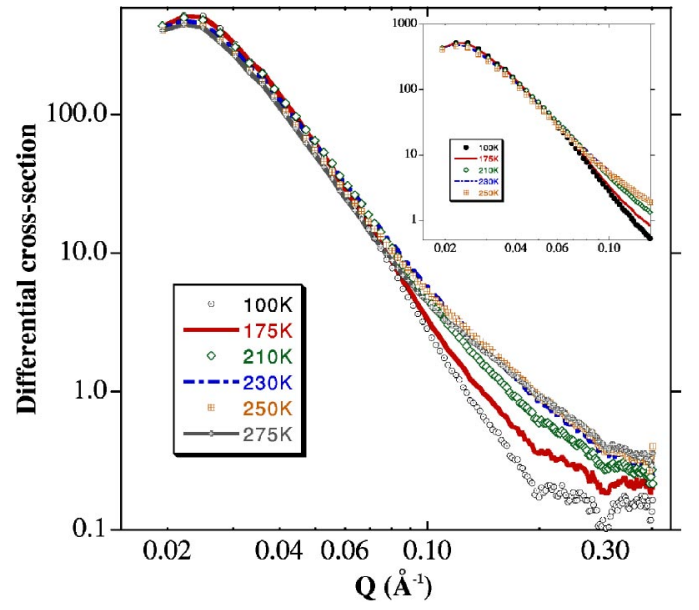

FIG. 1. A sample of the measured single-differential cross sections for $\mathrm{La}_{0.7} \mathrm{~Pb}_{0.3}\left(\mathrm{Mn}_{0.9} \mathrm{Fe}_{0.1}\right) \mathrm{O}_{3}$ for temperatures comprising the magnetic order-disorder transition that are given as insets. Absolute units are given as barns/sterad/formula unit. The inset displays a blowup for temperatures well below and above the ferromagnetic transition and low momentum-transfers.

dependence of the scattered neutron intensity at several momentum transfers. This is shown in Fig. 2(b) for four momentum transfers. There it is shown how a well-defined peak appears about $225 \mathrm{~K}$ for momentum transfers $Q \geqslant Q$ $=0.07 \AA^{-1}$. Its shape is reminiscent of what could be expected for a broad magnetic transition comprising a distribution of transition temperatures, and, in fact, the temperature range where such features develop nicely matches that where the magnetization curves displayed in Fig. 2(a) show their largest changes. The transition here observed is also followed at larger length scales, that is for $Q=0.03 \AA^{-1}$ by an increase in intensity indicative of the development of an ordered state.

To put the observations just referred to on quantitative grounds, and thus to derive pertinent values for the relevant physical quantities, we begin with considering that the measured differential cross section can be written as a sum of nuclear (structural) and magnetic contributions. Lower bounds to the size of the former entities are first provided by analysis of $\mathrm{x}$-ray diffraction patterns that set the grain sizes for the whole family of $\mathrm{La}_{0.7} \mathrm{~Pb}_{0.3}\left(\mathrm{Mn}_{1-x} \mathrm{Fe}_{x}\right) \mathrm{O}_{3}, \quad x$ $=0.1,0.2,0.3$, compounds within the interval 236-229 with increasing Fe percentage. A more accurate estimate of grain scattering was derived from a preliminary experiment carried out using the D22 small-angle scattering instrument of the I.L.L. ${ }^{12}$ The employed configuration allowed us to explore momentum transfers within the interval $0.007 \AA^{-1}$ $\leqslant Q \leqslant 0.07 \AA^{-1}$. Estimates for the size of entities giving rise to such scattering as derived from Guinier plots yielded values for particle radii $\bar{R}_{s}$ of about $360 \AA$. The values derived in such a study for samples with composition $\mathrm{La}_{0.7} \mathrm{~Pb}_{0.3}\left(\mathrm{Mn}_{0.8} \mathrm{Fe}_{0.2}\right) \mathrm{O}_{3}$ were in turn found to be compatible with results derived from a detailed full-polarized neutron diffraction study carried out on the D7 diffractometer at the I.L.L. ${ }^{12}$ There, a complete separation of magnetic and structural cross sections was achieved above some $90 \mathrm{~K}$, that is close to the midpoint of the macroscopic magnetic transition.

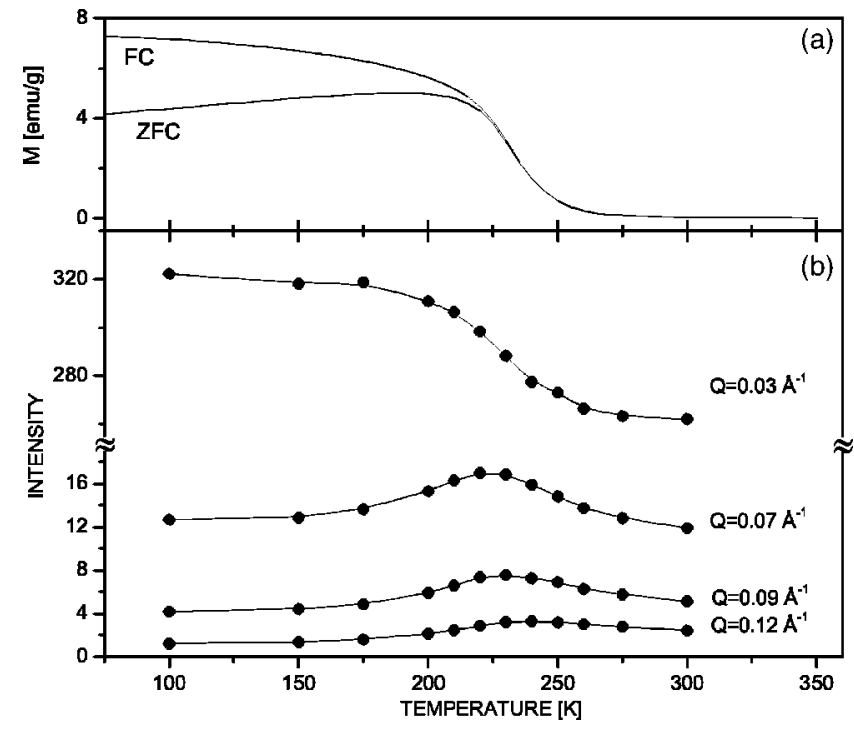

FIG. 2. (a) The zero-field cooling and field cooling magnetization curves measured under an applied $10 \mathrm{mT}$ field. (b) The temperature dependence of SANS intensity for selected values of the momentum transfer.

A strong ferromagnetic component that develops at lower temperatures hindered the measurements at lower temperatures. In all cases, the observed $Q$ dependence of the magnetic signal displayed a far milder decay than that for the structural counterpart. As an example, data for $T=200 \mathrm{~K}$, which is well above the macroscopic magnetic transition, yield ratios of structural/magnetic intensities of 0.50 for $Q$ $=0.5 \AA^{-1}$ and 0.35 for $Q=0.2 \AA^{-1}$, whereas for $T=90 \mathrm{~K}$ (midway in the transition) the figures for both wave vectors yielded 0.37 and 0.17 , respectively. On such grounds, we see that the structural component, attributable to large deffects, should become dominant for wave vectors of the order of $10^{-3} \AA^{-1}$, well beyond those explored in the present study.

Rather than following the steps of Refs. 6 and 7 that involve subtracting a high-temperature paramagnetic contribution, we model the scattering cross section as

$$
\begin{gathered}
\frac{d \sigma}{d \Omega}=\left.\frac{d \sigma}{d \Omega}\right|_{s}+\left.\frac{d \sigma}{d \Omega}\right|_{m} \\
\left.\frac{d \sigma}{d \Omega}\right|_{s}=A_{T} S_{s}^{\prime}(Q) P_{s}(Q) \\
\left.\frac{d \sigma}{d \Omega}\right|_{m}=A_{m} S_{m}^{\prime}(Q) P_{m}(Q)+\left[\frac{B}{Q^{2}+\left(\frac{1}{\xi}\right)^{2}}+\left(\frac{C}{Q^{2}+\left(\frac{1}{\xi}\right)^{2}}\right)^{2}\right],
\end{gathered}
$$

where subscripts $s$ and $m$ denote nuclear and magnetic contributions to the total cross section. It is written in terms of scattering from ensembles of interacting spherical particles of structural (chemical) origin with a shape determined by the form-factor $P_{s}$ and interference (diffraction) effects accounted for by the $S_{s}^{\prime}(Q)$ structure factor. In turn, the mag- 
netic component comprises $S_{m}^{\prime}(Q)$ and $P_{m}$ terms to account for interference effects between polydisperse magnetic particles and, finally, the Lorentzian plus squared-Lorentzian term included within the magnetic contribution account for paramagnetic fluctuations. ${ }^{13}$ The latter are specified in terms of the parameter $\xi$ that plays the role of a correlation length. ${ }^{14,13}$ The size distribution (polydispersity) of the particles ensembles is taken care of by ${ }^{15}$

$$
\begin{gathered}
S_{s, m}^{\prime}(Q)=1+\frac{\left|\left\langle F_{s, m}(Q)\right\rangle\right|^{2}}{\left\langle\left|F_{s, m}(Q)\right|^{2}\right\rangle}\left[S_{s, m}(Q)-1\right] ; \\
P_{s, m}(Q)=\left\langle\left|F_{s, m}(Q)\right|^{2}\right\rangle,
\end{gathered}
$$

which relates the apparent factors $S^{\prime}$ and $P$ to the individual $S$ and $F$ structure and form factors for interacting spheres of radius $R$.

Assuming that we are dealing with a smooth distribution of particle sizes the computation of the scattering cross section still requires the specification of particle and interparticle form factors as well as a distribution function for particle sizes. The latter is assumed to be given by a Gaussian function with mean $\bar{R}_{s, m}$ and deviation $\delta_{s, m}$.

Explicit forms for $F(Q)$ and $S(Q)$ factors are now needed. As regards the static structure factors $S(Q)$, the following assumptions are made. We first rely upon the wealth of previous evidence ${ }^{3,6,7}$ pointing towards a liquidlike distribution of droplets as the more apt description of the spatial distribution of such entities. Next, we use one of the simplest representations for the structure factor of a liquid as it is given by the Percus-Yevick integral equation for an ensemble of hard spheres ${ }^{16}$ (omitting the subscripts):

$$
\begin{gathered}
S(Q \sigma)=[1-n c(Q \sigma)]^{-1} \\
c(Q \sigma)=-4 \pi \sigma^{3} \int_{0}^{1} d s s^{2} \frac{\sin (s Q \sigma)}{s Q \sigma}\left(\alpha+\beta s+\gamma s^{3}\right) .
\end{gathered}
$$

where $\sigma$ is the hard-sphere diameter, $n$ is the number density of clusters, and $c(Q \sigma)$ is the direct correlation function in momentun space. Parameters $\alpha, \beta$, and $\gamma$ in the above equation are functions of a packing-fraction $\eta$

$$
\begin{gathered}
\eta=\frac{\pi}{6} n \sigma^{3} ; \quad \alpha=\frac{1+2 \eta}{(1-\eta)^{4}} ; \\
\beta=\frac{-6 \eta(1+\eta / 2)^{2}}{(1-\eta)^{4}} ; \quad \gamma=\frac{(1 / 2) \eta(1+2 \eta)}{(1-\eta)^{4}} .
\end{gathered}
$$

The particle form factor $F(Q)$ for a sphere of radius $R$ is given by ${ }^{15}$

$$
F(Q)=\left(\frac{4 \pi}{3}\right) R^{3}\left(\frac{3 j_{1}(Q R)}{Q R}\right),
$$

where $j_{1}$ stands for a spherical Bessel function.

Finally, because of the ferromagnetic nature of the ordered state, the onset of magnetic ordering will result in an intensity increase that will add up to peaks in the structural $S(Q)$. In consequence, we interpret the increase in intensity
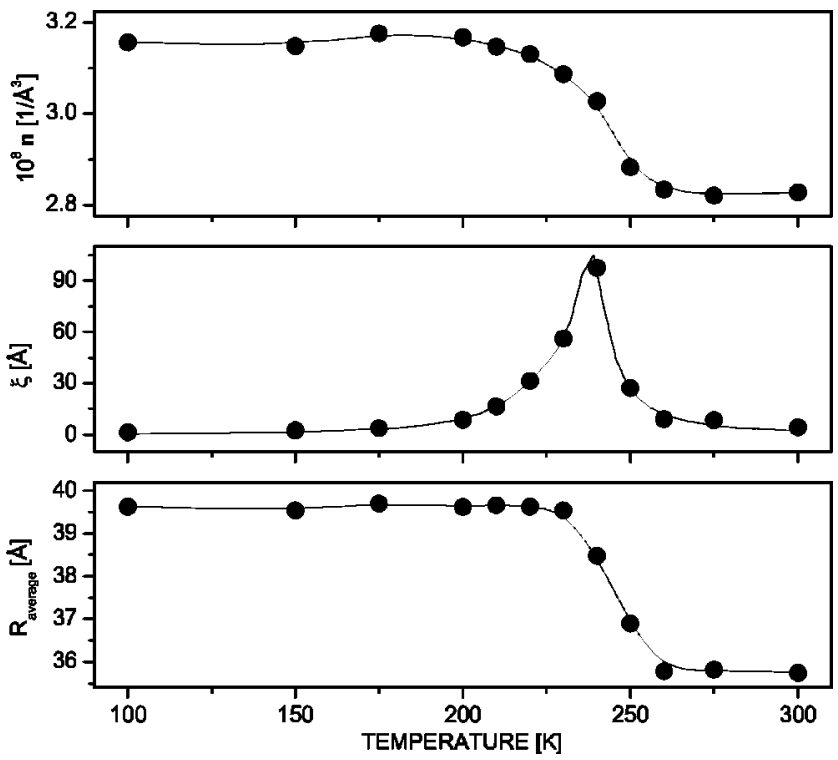

FIG. 3. The fitting parameters as a function of temperature. The upper frame displays the temperature dependence of the magnetic cluster number density $n$, the frame at the middle shows the correlation length associated to critical fluctuations, and the lower frame shows the radius of the average magnetic particle. Solid lines are drawn as guides to the eye.

for $Q=0.03 \AA^{-1}$ shown in Fig. 2(b) below $220 \mathrm{~K}$ as due to magnetic ordering and therefore $S_{m}(Q)$ accounts for such an intensity.

The experimental data displayed in Fig. 1 were then fitted parametrically using Eq. (1). In doing so we bear in mind that the changes observed in Fig. 2(b) are attributable in full to magnetic effects and therefore the structural contributions act as background terms. In addition, we have kept the dimensions of the structural clusters set to the value determined from small-angle neutron diffraction. ${ }^{12}$ The parameters required to complete the specification of $S_{s}^{\prime}$ were set to values such that $\sigma^{3}=8 \bar{R}_{s}^{315}$ and that for $\delta_{s}$ was set equal to that found to be optimal for the magnetic component. Using relatively large polydispersity values is required to avoid the appearance at larger $Q$ 's of marked oscillatory structures from the particle form factors, which are not observed experimentally.

An initial set of fits over the explored range of temperatures served to set the parameters concerning the magnetic particle-size distribution $f(R)$ to average values. This came as a consequence of the rather small variations found for some of the parameters over the full temperature range. The optimum, temperature-independent value for the hard-sphere radius $\sigma$ was found to be of 116(5) $\AA$ while the width of the particle distribution came out as $\delta=58 \AA$. The found value comes somewhat shorter than that derived from $\pi / Q_{p}$ $=125.5 \AA$ where $Q_{p}$ stands for the value of momentum transfers where the cross section shows its maximum. In contrast, the optimal values of the average radii of the magnetic particles were found to be significantly temperature dependent with bounds of $35.5 \AA$ for temperatures above the macroscopic magnetic transition and $39.5 \AA$ A below. Explicit results are shown in Fig. 3. 
The correlation length $\xi$ shown in Fig. 3 shows a peak centered about $235 \mathrm{~K}$ that is accompanied by a sharp rise and fall of the ratio between magnetic and structural contributions to the cross section (not shown). Both pieces of information serve to quantify the magnetic transition into a ferromagnetically ordered phase already discovered in the raw cross-section data.

The parameters associated with intercluster interactions, such as the number density of clusters $n$ and the average radius $\bar{R}$, show a contrasting behavior with respect to those characterizing the paramagnetic fluctuations. Both $n$ and $\bar{R}$ show a pronounced step at temperatures where $\xi$ and the ratio of magnetic to total intensities show a maximum. Such a stepping behavior constitutes a clear signature of an ordering transition mediated by entities with radii of about $35-40 \AA$. The increase in packing fraction translates into an increase in the magnetic contribution to the peak of $S(Q)$ that shows up at $Q_{p} \approx 0.025 \AA^{-1}$. Such a wave vector marks an average separation between such clusters, which corresponds to length scales well above those found for paramagnetic spin-fluctuations (see Ref. 9). The magnitude of the cluster radii is certainly larger than that of $17-20 \AA$ reported in preliminary studies on undoped manganites, ${ }^{4,6}$ but are smaller than those recently reported by Caspi et al. ${ }^{6}$ for a two-electron-doped system or the large micron-sized domains probed by Sarma et al. by photoelectron spectroscopy. ${ }^{3}$

Our results thus display two distinct behaviors depending upon the characteristic lengths involved. At short length scales paramagnetic spin fluctuations incompletely freeze into an ordered state, a process that is accompanied by the development of strong interactions between the larger clusters. A pure ferromagnetic metallic state, however, never reaches complete stabilization as proven by previous Mössbauer data, ${ }^{10}$ a phenomenon now suspect to be due to disorder. ${ }^{17}$

We indeed observe that the maximum ferromagnetic cluster size ( $\approx 80 \AA$ in diameter) remains well below the crystallite size. Whether this comes as a consequence of an incipient frustration built in by the Fe doping, the effects of quenched disorder, or else to the gradual emergence of an orbitally polarized antiferromagnetic state cannot be resolved with the means at our disposal.

Finally, and to provide a sense of scale, a comparison of the cluster radii here reported on with characteristic (ferro-) magnetic grain sizes can also be given. These have been estimated from the blocking temperatures of the ZFC-FC curves as well as from recent $\mu \mathrm{SR}$ data and yield values within the interval $15-21 \mathrm{~nm},{ }^{18}$ which are somewhat below the lower bound found for the crystallographic grain size.

In summary, our neutron data have proved the presence of a spatial distribution of magnetic droplets. These remain as well-defined entities well into the ferromagnetic state where they coexist with smaller regions which, once ordered, give rise to the long-ranged ordered state explored by conventional diffraction means. ${ }^{8}$
*Fellow of CONICET, Argentina.

†Electronic address: jbermejo@we.lc.ehu.es

*C.S.I.C., Inst. Estructura de la Materia, Serrano 123, E-28006 Madrid, Spain.

${ }^{1}$ E. Dagotto, Nanoscale Phase Separation and Colossal Magnetoresistance (Springer-Verlag, Berlin, 2002).

${ }^{2}$ G. Van Tendeloo et al., Rep. Prog. Phys. 67, 1315 (2004); J. M. D. Coey et al., Adv. Phys. 48, 167 (1999); E. Dagotto et al., Rep. Prog. Phys. 344, 1 (2001).

${ }^{3}$ D. D. Sarma et al., Phys. Rev. Lett. 93, 097202 (2004); C. D. Ling et al., Phys. Rev. B 68, 134439 (2003); Ch. Simon et al., Phys. Rev. Lett. 89, 207202 (2002); G. Biotteau et al., Phys. Rev. B 64, 104421 (2001); W. Tong et al., Phys. Rev. B 70, 014422 (2004); E. V. Mostovshchikova et al., Phys. Rev. B 70, 012406 (2004).

${ }^{4}$ J. M. De Teresa et al., Nature (London) 386, 256 (1997); P. G. Radaelli et al., Phys. Rev. B 63, 172419 (2001); P. A. Algarabel et al., ibid. 65, 104437 (2002).

${ }^{5}$ D. N. Argyriou et al., Phys. Rev. Lett. 89, 036401 (2002).

${ }^{6}$ M. Hennion et al., Phys. Rev. Lett. 81, 1957 (1998); E. N. Caspi et al., Phys. Rev. B 69, 104402 (2004).

${ }^{7}$ E. Granado et al., Phys. Rev. B 68, 134440 (2003).

${ }^{8}$ J. Gutiérrez et al., Phys. Rev. B 61, 9028 (2000).

${ }^{9}$ J. Gutiérrez et al., J. Magn. Magn. Mater. 272-276, e983 (2004).

${ }^{10}$ J. M. Barandiarán et al., J. Phys.: Condens. Matter 14, 12563 (2002).

${ }^{11}$ M. A. Howe et al., Correct (computer code), NFL Studsvik (1996).

${ }^{12}$ P. P. Romano, Inst. Laue Langevin Report of Stage Activity, Grenoble, 2003.

${ }^{13}$ R. J. Birgeneau et al., Phys. Rev. B 28, 1438 (1983).

${ }^{14}$ S. Lovesey, Theory of Neutron Scattering from Condensed Matter (Oxford Science Publications, Oxford, 1984), Vol. 2, Chap. 13.

${ }^{15}$ M. Kotlarchyk and Sow-Hsin Chen, J. Chem. Phys. 79(5), 2461 (1983).

${ }^{16}$ N. W. Ashcroft and J Lekner, Phys. Rev. 145, 1 (1966).

${ }^{17}$ Y. Motome et al., Phys. Rev. Lett. 91, 167204 (2003); C. Sen et al., Phys. Rev. B 70, 064428 (2004).

${ }^{18} \mathrm{~J}$. Gutiérrez et al. (unpublished). 